Revue des patrimoines

$36 \mid 2018$

Les archives photographiques de presse, pratiques comparées et enjeux méthodologiques

\title{
« Ne pas laisser s'effacer l'histoire de ceux qui la racontent ». Le cas de l'Association de préfiguration de la Fondation Patrick Chauvel
}

'Don't lose the history of those who made it', the Patrick Chauvel Foundation

\section{Émilie Fromentèze}

\section{OpenEdition}

Journals

Édition électronique

URL : http://journals.openedition.org/insitu/18413

DOI : $10.4000 /$ insitu. 18413

ISSN : 1630-7305

Éditeur

Ministère de la culture

Référence électronique

Émilie Fromentèze, « « Ne pas laisser s'effacer I'histoire de ceux qui la racontent ». Le cas de

I'Association de préfiguration de la Fondation Patrick Chauvel », In Situ [En ligne], 36 | 2018, mis en

ligne le 18 octobre 2018, consulté le 02 mai 2019. URL : http://journals.openedition.org/insitu/18413 ; DOI : 10.4000/insitu. 18413

Ce document a été généré automatiquement le 2 mai 2019.

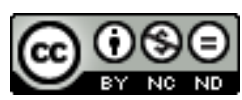

In Situ Revues des patrimoines est mis à disposition selon les termes de la licence Creative Commons Attribution - Pas d'Utilisation Commerciale - Pas de Modification 4.0 International. 
« Ne pas laisser s'effacer l'histoire de ceux qui la racontent ». Le cas de l'Association de préfiguration de la Fondation Patrick Chauvel

\author{
'Don't lose the history of those who made it', the Patrick Chauvel Foundation
}

Émilie Fromentèze

"On ne savait pas!"

Cette phrase entendue à la fin de la Seconde Guerre mondiale n'est plus possible. Aujourd'hui, on sait. Les journalistes sont partout épaulés par des « reporters citoyens ". L'information circule dans le monde entier par des réseaux de toutes sortes, rapide et en temps réel.

Une technologie fabuleuse au service de l'information, qui a changé les moyens de travailler.

Transmettre rapidement, c'est un plus, mais cela n'exclut pas de prendre son temps pour observer, s'imprégner des événements afin de mieux comprendre et raconter. Le défi pour les journalistes qui sillonnent le monde est de rester des témoins passionnés qui prennent le temps d'être à l'écoute. Trouver la bonne distance, pour photographier.

«La première victime de la guerre est la vérité! " C'est à nous de la rechercher, de la trouver et de la montrer. Notre pouvoir n'étant que la moyenne de ce que l'on peut faire et de ce que les circonstances nous permettent. En zone de guerre, cet accord de notre propre force avec la fatalité des événements est fragile. Une mission difficile, on est dans "l'Histoire bataille", 
et nos témoignages feront partie de la mémoire

collective.

Une lourde responsabilité qui est aussi une chance formidable, celle d'avoir un objectif clair. Être celui à qui les hommes en colère confient leur détresse. Partager leurs épreuves pour ensuite raconter à ceux qui veulent savoir. Connecter le monde de la paix avec celui de la guerre, pour que les hommes décident de leur destin en connaissance de cause.

Une passion, une façon de vivre que j'aimerais transmettre pour ne plus entendre: " On ne savait pas! ». Aujourd'hui, ceux qui ne savent pas sont ceux qui ne veulent pas savoir... Patrick Chauvel ${ }^{1}$

\section{Introduction}

Aujourd'hui, les archives issues de la presse, notamment les photographies, ont acquis une certaine valeur sur le marché de l'art. Mais plus que leur valeur monétaire, c'est la valeur historique de ces fonds qui intéresse les archivistes. De plus en plus de photographes se posent la question du devenir de leur travail parce que, comme le rappelle le cinéaste Pierre Schoendoerffer dans la préface de l'ouvrage Rapporteur de guerre $^{2}$, citant un poète ${ }^{3}:$ "L'humanité qui perd sa mémoire est condamnée à mourir de froid. » Il y a urgence à préserver et à présenter ces morceaux d'histoire dont les photoreporters ont été les témoins et les collecteurs. Par les photographies qu'ils ont prises, ils rendent compte de l'évolution du monde, en particulier à travers ses conflits.

Patrick Chauvel est l'un d'entre eux. Accompagnant les soubresauts de ce monde depuis près de cinquante ans, ce photographe, considéré aujourd'hui comme l'un des plus grands reporters français, a documenté et témoigné ; il a vécu jusque dans sa chair les conflits, parfois au péril de sa vie. De ses missions à travers le monde en guerre, il a rapporté des dizaines de milliers d'images qui ont été publiées dans la presse. Aujourd'hui, sans quitter pour autant le domaine de l'illustration immédiate, il propose de donner un sens nouveau à ses archives et de mettre au jour de nombreux épisodes de l'histoire contemporaine, « pour ne pas oublier».

Acceptant la proposition de mécènes suisses qui ont décidé de financer cette aventure, Patrick Chauvel a fondé en mars 2014 l'Association de préfiguration de la Fondation Patrick Chauvel dont le but est de réunir, archiver, valoriser et promouvoir l'ensemble de son œuvre. Cette initiative est d'autant plus intéressante que son auteur, qui en est aussi le principal contributeur, est encore là pour retracer sa carrière au travers de ses archives, qu'il a rassemblées, et autour desquelles il peut témoigner.

La volonté de préserver ses archives est une idée qui tient à cœur à Patrick Chauvel. Mais trouver un lieu de conservation disposé à accueillir des archives de presse et, singulièrement, des archives de photoreporter, n'est pas chose facile. Ces dernières sont en effet souvent volumineuses, leur classement parfois succinct ou inexistant, les informations qui les accompagnent, approximatives; en outre, les questions de droits doivent être prises en compte, et la gestion des ventes et de la valorisation respectée. Grâce au mécénat, Patrick Chauvel s'est lancé, avec une équipe qu'il a lui-même 
constituée, dans cette entreprise lourde, chronophage, coûteuse et nécessitant un investissement humain important.

Le travail archivistique effectué au sein de l'Association de préfiguration de la Fondation Patrick Chauvel repose sur la mise en place d'un système de traitement appliqué au fonds spécifique d'un photoreporter. Il sera complété par la rédaction d'un vade-mecum sur le traitement et la conservation des photographies de presse et autres documents ou objets de travail dont l'importance pour l'histoire collective est sous-estimée.

\section{Patrick Chauvel, un reporter engagé dans la profession}

«Je serre mes films dans ma poche. Il faut que je les montre ${ }^{4}$.» C'est sur ces mots que se termine l'un des ouvrages de Patrick Chauvel, reporter au long cours. Un trait familial puisqu'avant lui, son père, Jean-François Chauvel (1927-1986), grand reporter, et son grand-père, Jean Chauvel (1897-1979), diplomate et ambassadeur de France, ont eu ce goût d'ailleurs, qu'ils lui ont transmis. Passionné par les récits de son père mais aussi de son oncle Pierre Schoendoerffer et de leurs amis Joseph Kessel, Lucien Bodard et Gilles Caron $^{5}$, à 17 ans, il décide de partir pour la première fois sur un terrain de guerre. Plus qu'un métier, c'est un engagement, selon les termes du photographe James Nachtwey :

$\mathrm{Au}$ début, c'est par goût d'aventure, tu veux te tester, voyager, faire carrière, et aussi faire la différence. Et plus tu es confronté à la souffrance des autres, à l'injustice et à la tragédie, plus tes raisons personnelles s'effacent naturellement devant l'envie de témoigner et d'essayer de maintenir le dialogue afin que les choses changent. Si personne ne sait ce qui se passe, si tout se fait dans l'ombre, tout peut arriver ${ }^{6}$.

Patrick Chauvel entre au journal France-Soir comme stagiaire et dès lors, il ne pense qu'à partir couvrir les conflits à l'étranger. Ses premiers salaires lui permettent de réaliser ses premiers reportages, notamment sur la guerre des Six Jours (1967). Pigiste pour Associated Press et l'agence de presse Reuters dans leurs bureaux de Saïgon pendant la guerre du Vietnam, il entre à Sipa Press puis Sygma dans les années 1970.

Il est de ces rares photoreporters encore en vie à avoir couvert la guerre des Six Jours, mais aussi les événements du Vietnam, du Cambodge, d'Irlande du Nord, d'Iran, du Liban, du Panama, du Nicaragua, d'Afghanistan, de Tchétchénie, de Libye, etc. ${ }^{7}$ Chaque fois, il va au plus près de la réalité, au-delà de la simple actualité. Ses reportages dépassent les histoires anecdotiques pour assigner l'Histoire et l'Humanité. L'intégralité de son travail témoigne de cinquante années d'histoire mondiale, près d'un demi-siècle de mémoire.

Ses images sont publiées dans les plus grands journaux, de Paris Match à Times Magazine, Life ou encore Newsweek, et exposées dans le monde entier. Pour une photographie du massacre de San Salvador en $1980^{8}$, il obtient le prix Missouri de l'Université de journalisme des États-Unis (1984). En 1996, son travail sur le conflit en Tchétchénie remporte un des prix du prestigieux concours World Press Photo et le prix du Festival international du scoop et du journalisme d'Angers.

En Tchétchénie, il décide d'adjoindre la parole à l'image et utilise désormais le documentaire pour exprimer ce qu'il comprend des conflits. Mais il ne lâche pas pour autant son appareil photo et offre des images fixes d'une réalité qu'il peut maintenant aussi montrer comme se déroulant sous ses yeux. Il réalise ainsi une série de documentaires engagés pour la télévision française : la violence faite aux femmes en 
Algérie, le conflit israélo-palestinien, les traumatismes des enfants tchétchènes, le désarroi des artistes irakiens, les violences au Pakistan, en Thaillande, à la frontière de l'Afghanistan, etc. ${ }^{9}$ En 1998, il réalise avec Antoine Novat le documentaire Rapporteurs de guerre, dans lequel il interroge des reporters de guerre sur les raisons de leur engagement dans ce métier. Au début des années 2000, Patrick Chauvel commence à écrire et raconte notamment son engagement dans un récit qui en reprend le titre ${ }^{10}$.

En octobre 2009, invité à présider le Festival des correspondants de guerre de Bayeux, il présente une exposition intitulée «Guerre-ici» présentant des photomontages combinant des photographies prises dans des zones de conflits et des monuments emblématiques parisiens. Réalisées avec l'aide de Paul Biota pour le montage, ces images sont des projections de la guerre à Paris, et cherchent à alerter ceux qui se moquent des guerres en les imaginant loin d'eux.

Très impliqué dans la profession, il se place dans la transmission des valeurs et de l'éthique du métier de photoreporter en participant à de nombreuses conférences et tables rondes sur le sujet. Il donne également des cours de photojournalisme dans diverses institutions et anime des workshops sur la photographie, le journalisme, la mémoire et la guerre à travers le monde, formant ainsi de jeunes reporters.

Patrick Chauvel est l'un des co-fondateurs de la Fondation WARM ${ }^{11}$ qui travaille sur la mémoire des guerres contemporaines dans le monde et s'engage avec des chercheurs d'horizons divers dans une réflexion sur la guerre et la résolution des conflits.

En 2014, le photoreporter crée donc une structure destinée à rassembler l'ensemble de son travail, à construire une plateforme de réflexion sur le métier de reporter de guerre et à servir de relais entre les générations en mettant en avant le travail de photographes peu connus. Il a souhaité ouvrir ses archives à d'autres photographes. Dans un premier temps, il a associé des photographes vietnamiens, «Ceux du Nord $»^{12}$, par le biais d'un projet photographique singulier. Une exposition puis un livre ont permis de présenter face à face des photographies de la guerre du Vietnam prises par les Vietnamiens et par lui-même. Les photographies prises par ses confrères vietnamiens ont ensuite été incorporées à l'association et peuvent continuer à être présentées. Dans un second temps, Patrick Chauvel souhaite que cette structure assure la conservation de fonds d'autres photoreporters.

\section{Fondations de reporters : photographie d'un projet}

Ces dernières années s'est développée une conscience internationale de l'histoire et de la mémoire de l'Humanité. En relation avec cette tendance, les reporters, de plus en plus soucieux du devenir de leurs archives, recherchent des structures qui permettent à la fois la conservation de leurs archives dans de bonnes conditions et la valorisation et la diffusion de leurs images. Certains se rapprochent d'institutions publiques, centres d'archives, bibliothèques ou musées, qui peinent à répondre favorablement à leurs demandes, pour des raisons aussi diverses que l'ampleur des fonds ou la gestion des droits. Le sort des agences qui ferment ne fait qu'accroître l'inquiétude des photographes, qui voient leurs archives menacées de disparition. Le Centre international de photojournalisme (CIP), dont la création a été annoncée en $2015^{13}$, pourrait apparaître comme une proposition à la reprise de ces fonds. Si la note d'intention énonce clairement les ambitions du projet, la création elle-même et la gestion de l'institution restent à ce 
jour très floues, ce qui n'en fait pas encore, pour de nombreux reporters, un lieu vers lequel se tourner.

Sans solution, certains photographes gèrent leurs archives eux-mêmes et en assurent la diffusion, d'autres créent leurs propres structures, de nature et de statut différents. Citons la Fondation Henri Cartier-Bresson, l'une des plus connues, instaurée par Henri Cartier-Bresson lui-même. Elle a vu le jour en mai 2003 et conserve aujourd'hui les fonds du photographe et de Martine Frank, son épouse. Fondation privée reconnue d'utilité publique, elle est financée par divers mécènes privés. Elle a obtenu une reconnaissance légale aux États-Unis en raison de sa dimension internationale, ce qui assure un plus grand rayonnement à l'institution et à l'œuvre.

Les objectifs de cette structure sont divers : conserver et préserver un fonds patrimonial majeur pour le valoriser et le rendre accessible aux chercheurs ${ }^{14}$.

Le statut de fondation, pour une petite structure, reste assez exceptionnel en France, notamment pour des raisons financières puisqu'un apport de 1,5 million d'euros est nécessaire pour que l'État lui reconnaisse le caractère d'utilité publique ${ }^{15}$. En Suisse, l'apport demandé n'est que de 200000 euros. C'est ainsi que la Fondation Gilles Caron a été créée en 2007 à Genève par sa veuve, Marianne Caron-Montely. Son principal objectif est de « donner à Gilles Caron la place qui lui revient dans l'histoire du journalisme, de l'art et de la photographie $»^{16}$.

Pour le moment la structure de base est une association de préfiguration d'une fondation, mais Patrick Chauvel a d'ores et déjà réfléchi à la potentialité de créer une structure plus ambitieuse. Ayant pour vocation première de réunir, archiver, valoriser et promouvoir l'ensemble de don œuvre, la structure a pour but d'évoluer afin d'ajouter, à la sauvegarde de ce patrimoine, la possibilité d'ouvrir cette importante source d'information, aux chercheurs et universitaires, et ainsi, développer des pistes de réflexion sur l'histoire contemporaine et internationale. D'autres actions de valorisation sont envisagées, comme l'organisation de rencontres et d'expositions. Elle se donne également pour objectif de défendre et promouvoir l'éthique du reportage dans les zones de conflits et de contribuer à la formation de jeunes reporters.

La Fondation WARM est une autre structure qui, elle, « a pour but de rassembler au sein d'une fondation les travaux de tous les journalistes, de tous les artistes et de tous les écrivains de qualité qui travaillent sur les conflits contemporains $»^{17}$. Créée le 6 avril 2012, a pour objectif, à terme, d'ouvrir un centre permettant de recueillir et «collecter la mémoire des conflits postérieurs à la guerre du Vietnam et de numériser ces archives ${ }^{18}$. Actuellement, elle est installée dans plusieurs villes (Sarajevo, Paris et Genève), et a le statut de « fonds de dotation » en France, ce qui lui permet de financer d'autres projets en lien avec ses missions.

Le statut d'association donne la possibilité de débuter et d'investir dans les moyens humains et le matériel de travail. Le dessein étant de pouvoir faire vivre la structure de manière autonome et de transformer ensuite l'association en fondation afin de lui donner un peu plus d'importance et de crédit.

Le statut de fondation, en France, apporte également une garantie de l'État, ce qui n'est pas le cas d'un fonds de dotation. Si la fondation fait faillite, l'État sera garant des fonds collectés, et cette garantie favorise le mécénat. Étant garant des fonds conservés, en cas de disparition de la fondation, l'État en devient propriétaire. Ainsi, les mécènes investissent dans la conservation et la valorisation des fonds, pas dans une structure. 
Mais plus que son statut, ce sont les fonds qu'une structure conserve, ses objectifs et ses missions, qui importent. Le statut n'est qu'un moyen parmi d'autres de permettre ces réalisations.

\section{Sauvegarder la mémoire}

Les archives de la presse écrite conservent la mémoire des événements passés. À la fois écrite et photographiée, l'histoire qu'elles recèlent est celle d'un peuple, d'un pays, d'une région, voire de plusieurs. Elle peut être locale, nationale ou mondiale. Ces archives peuvent être conservées dans une agence de presse, un journal ou même tout simplement chez leur producteur. Mais elles demeurent ce que l'on nomme plus communément des archives d'entreprises.

Dans le cas qui nous intéresse, le domaine de la presse, il produit des objets, tirages, diapositives, négatifs, destinés à être publiés. Ces objets ne sont pas considérés comme patrimoniaux à première vue et sont avant tout des documents de travail. Alors quel est l'intérêt de sauvegarder de telles archives à l'heure actuelle?

\section{La récupération des images}

À l'âge d'or du photojournalisme, les photographes faisaient des photos sans se préoccuper, la plupart du temps, de leur devenir ; Patrick Chauvel n'a pas échappé pas à la règle, et retrouver et rassembler l'ensemble de sa production a été une opération délicate.

La première source de récupération, ce sont les archives personnelles de Patrick Chauvel : ses disques durs, où sont rangés les reportages réalisés en numérique depuis les années 2000, mais aussi la cave de son appartement parisien, pour les documents analogiques. Durant près de vingt ans, quatre malles américaines remplies de diapositives, négatifs et tirages y avaient été entreposées, faute de place, dans le froid et l' humidité.

La seconde source, l'agence Corbis, qui conservait une grande partie du matériel photographique produit par Patrick Chauvel alors qu'il travaillait pour l'agence Sygma, entre 1975 et 1996. Dès le démarrage de son projet, Patrick Chauvel a entamé des négociations auprès des différentes institutions détenant du matériel susceptible de lui appartenir, dont le bureau français de la société américaine Corbis-Sygma, ainsi dénommée après le rachat de Sygma par Corbis en 1999. Un accord de restitution a pu être conclu, et pendant près de deux ans, des cartons contenant le nectar photographique ont rejoint les bureaux de l'association, petit à petit. La société Corbis-Sygma, après sa mise en liquidation, a subi de nombreux licenciements et ne disposait donc plus de personnel pour assurer rapidement ce transfert (fig. 1). 


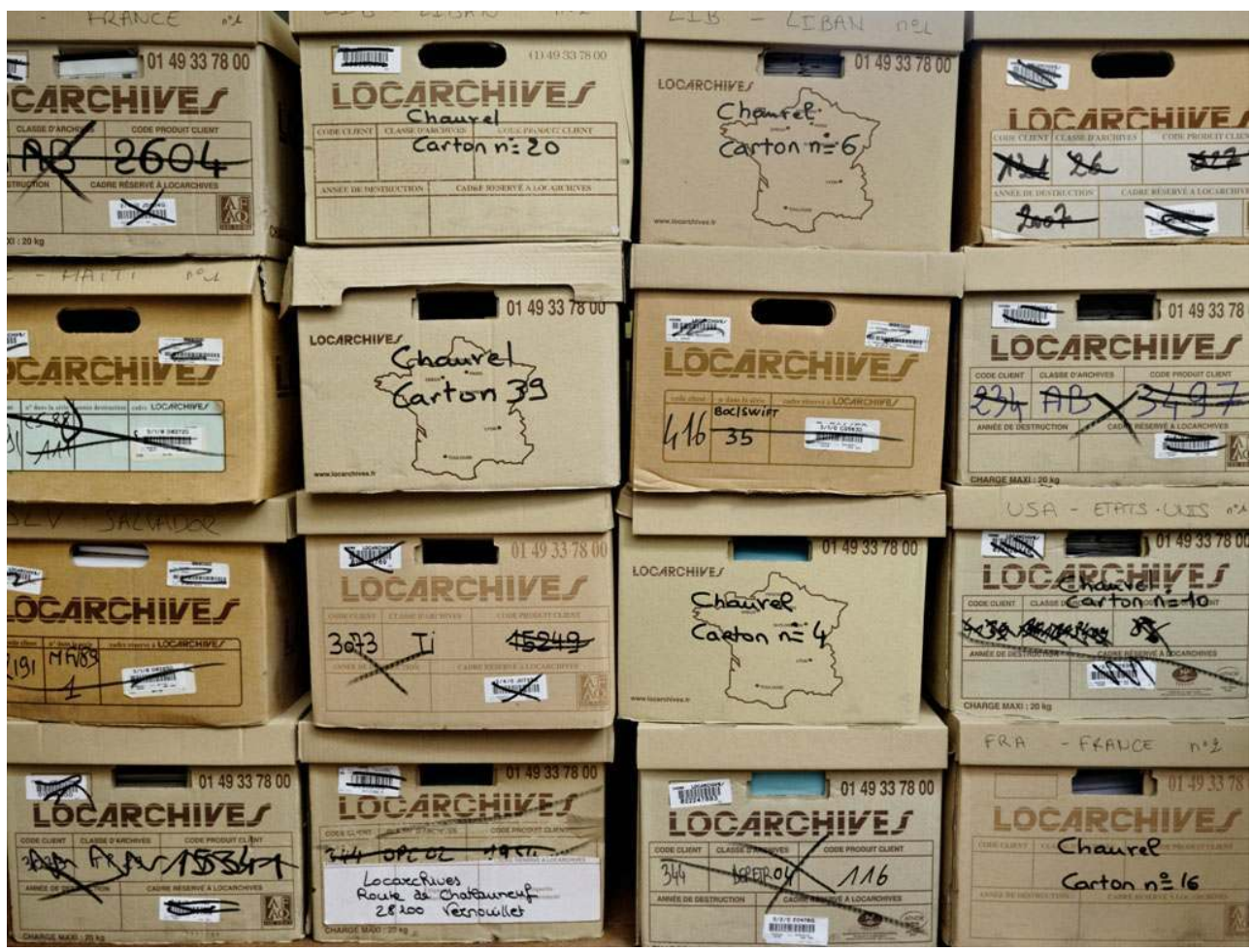

Cartons contenant les rendus de Corbis-Sygma provenant du local Locarchives de Garnay.

(c) Gaëlle Girbes.

Pendant que cette restitution avait lieu, en janvier 2016, Bill Gates a vendu le fonds photographique Corbis-Sygma à Unity Glory International, une filiale de l'entreprise de médias chinoise Visual China Group (VCG) ${ }^{19}$. Ces fusions ou rachats complexifient encore pour les photographes l'accès à leurs archives, diffusées aujourd'hui par des sociétés qui n'ont qu'une connaissance très limitée, voire aucune, des stocks qu'elles récupèrent.

On mesure ici à quel point l'intervention de l'association et la volonté de réunir un fonds de l'ampleur de celui de Patrick Chauvel ont été déterminantes. S'il a pu récupérer ses archives, cela n'a pas été le cas de nombreux photographes, pourtant désireux de le faire.

De nos jours, les agences photographiques vendent les images à très bas prix, souvent quelques centimes. Le développement des technologies numériques et l'économie actuelle donnent accès à des contenus photographiques à moindres frais (ou coûts). C'est un vrai problème pour les photographes de métier qui travaillent en agence. $\mathrm{Si}$, auparavant, ils rencontraient des difficultés à en tirer des revenus suffisants, à faire produire les reportages avant de partir, leurs difficultés se sont accrues. Les agences ont à leur disposition de très nombreuses images et plusieurs photographes pour un même événement. Elles vendent les images quelques centimes pour alimenter les contenus médiatiques numériques, et ce qui revient aux photographes peut ne pas dépasser quelques dizaines d'euros par mois.

La volonté de certains photographes de sortir de cette économie qui les paupérise tout en diffusant leurs images autrement et de manière plus complète est une des conséquences de cette mutation. Patrick Chauvel connaît ses images, et même si elles n'ont pas été 
publiées, il est capable de retrouver un cliché, un reportage inédit sur un sujet historique, et d'en assurer lui-même la valorisation et la diffusion.

\section{Tri et traitement du fonds}

Le traitement du fonds a débuté par ce que conservait le photographe. Parmi ses images, il a fallu mettre de côté les éléments trop dégradés, attaqués par les moisissures et l'humidité présente dans les différents lieux de stockage.

Par la suite, après l'arrivée des premiers cartons d'archives en provenance des entrepôts loués par Corbis-Sygma auprès de la société Locarchives à Dreux, nous avons mis en place une première procédure de traitement des documents qui a évolué au fil de la restitution des cartons, généralement au rythme de deux cartons par mois. Il s'agissait de négatifs souples, de diapositives, de tirages argentiques, d'objets, de carnets de notes, de coupures de presse, de films, de fichiers numériques, etc. arrivés « en vrac », qu'il a d'abord fallu trier par support puis de manière topographique. Ce classement a été élaboré pour s'adapter à la production. Les images étant produites dans le cadre de reportages sur les conflits mettant aux prises plusieurs pays, mais se déroulant souvent sur un territoire précis.

Ayant commencé à trier les documents par support et par pays, il a souvent été fastidieux et long de reconstituer les reportages. La plupart du temps, l'exercice s'est transformé en un jeu de piste qui progressait en fonction des cartons rendus dans lesquels on pouvait trouver, parfois un reportage complet, plus souvent quelques images qui venaient compléter celles que nous avions déjà retrouvées ou commencer une nouvelle série. Les images sélectionnées par l'agence pour la diffusion, les fameux « points rouges » côtoyant dans les cartons les « non choix », nous ne pouvions pas savoir devant quel cas nous nous trouvions, et les employés de Corbis-Sygma, l'ignorant tout autant, ne pouvaient pas nous aider dans cette reconstitution. Un premier rangement a donc précédé la reconstitution d'un ordre de reportage (un rassemblement des différents duplicatas des images et une mise en ordre des événements).

Le lien entre les différents éléments relatifs à un même reportage est maintenu grâce à l'instrument de recherche qui décrit par reportage la nature des documents présents.

Les documents ont été reconditionnés dans des boîtes d'archives. Sur chaque boîte ont été reportés les numéros d'inventaire des reportages qu'elle contient. À l'intérieur, les reportages ont été placés dans des pochettes sur lesquelles sont indiqués l'ancien numéro de reportage et le nouveau numéro d'inventaire (fig. 2). 
Figure 2

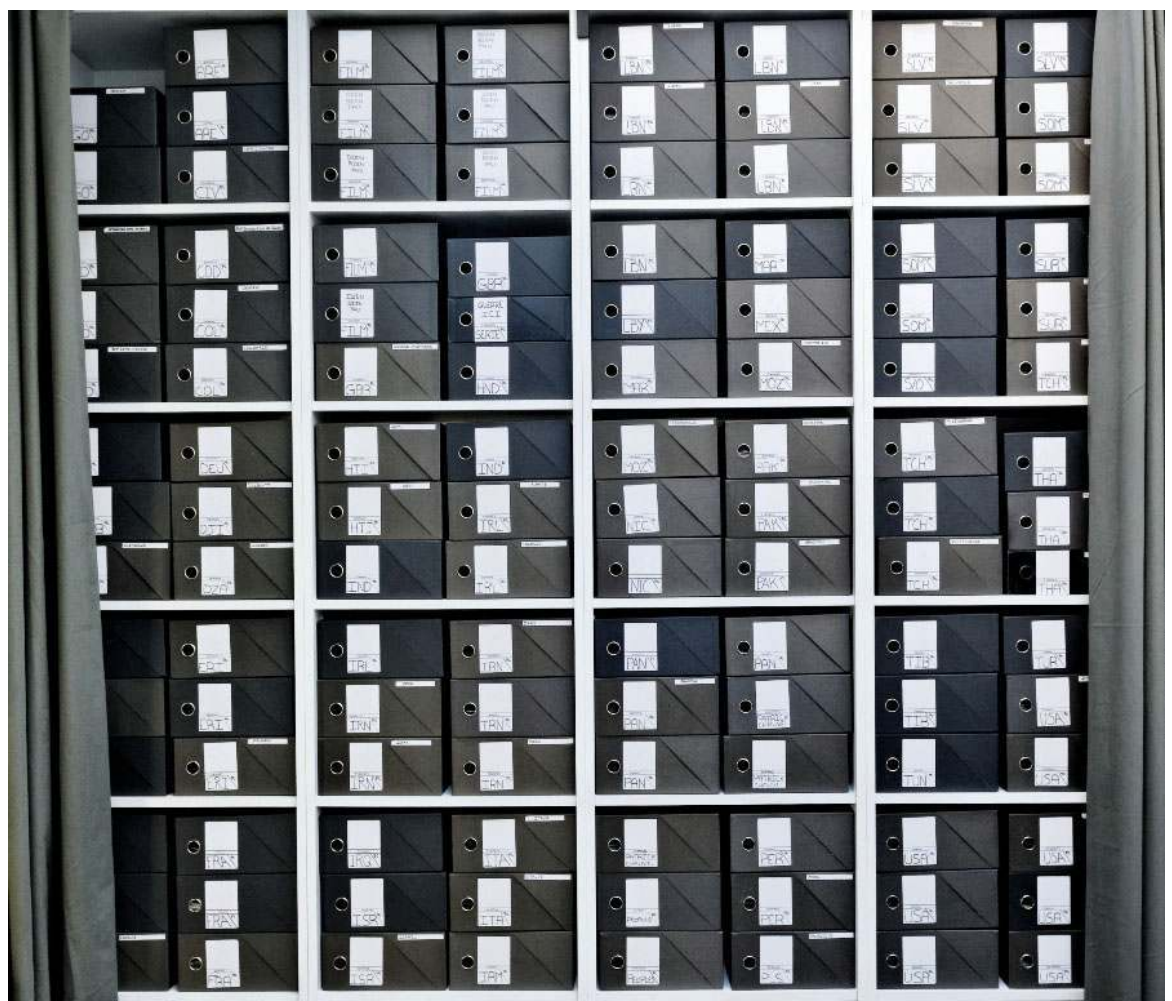

Vue de la photothèque physique après reconditionnement du matériel.

(C) Association de préfiguration de la Fondation Patrick Chauvel.

Le tri des documents numériques, dont la quantité est considérable, a été plus complexe. Il s'est agi en fait de distinguer les photographies originales en fichier numérique natif des reproductions, parfois nombreuses, de photographies analogiques dont il fallait les rapprocher. Une image peut être ainsi présente de multiples fois, sous différentes formes ou supports (CD, clefs USB, disques durs...) avec des métadonnées, des qualités et des formats différents. Une même image peut ainsi être en basse ou haute définition, avec ou sans champ IPTC ${ }^{20}$ renseigné, retouchée ou non, etc., ce qui nécessite un tri qui ne peut se faire qu'image par image, en vérifiant soigneusement chacune des informations afin de conserver et archiver les images de qualité. Cette opération a fait apparaître quelques numérisations de documents argentiques anciennes qui ne pouvaient plus être utilisées en l'état, obligeant à entreprendre de nouvelles numérisations (fig. 3). 


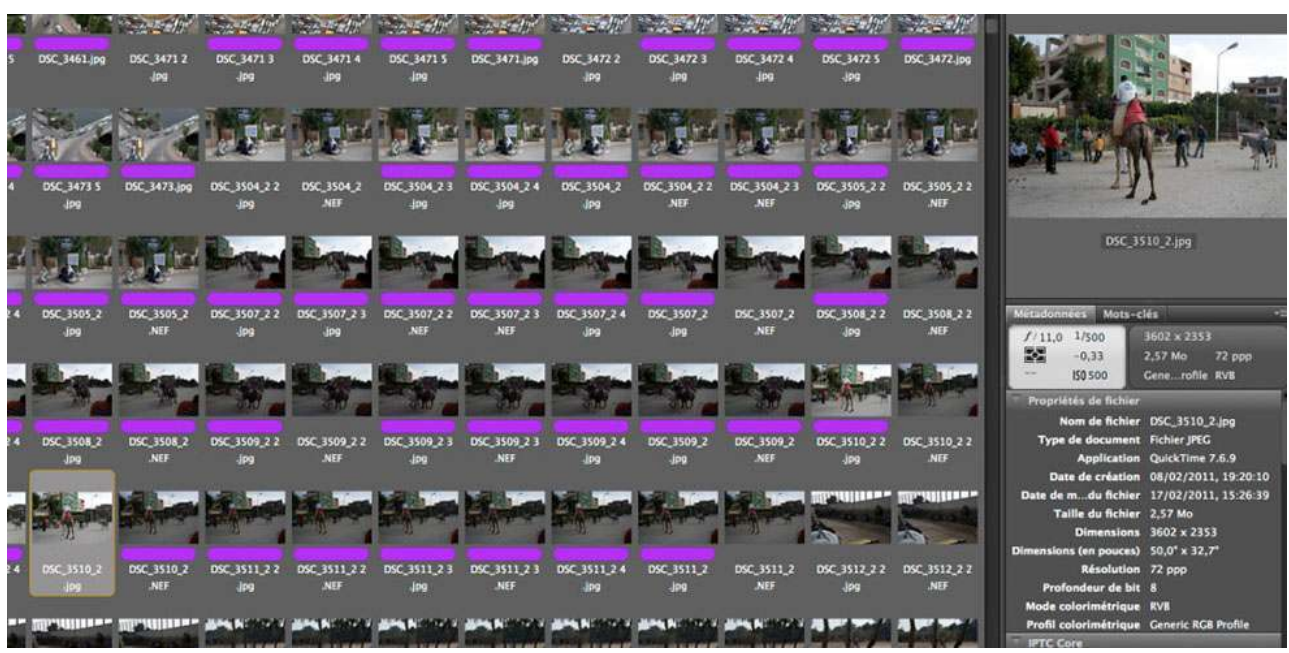

Extrait du tri numérique des images sur le logiciel Bridge.

(c) Association de préfiguration de la Fondation Patrick Chauvel.

Pour mettre en place le plan de classement, il a fallu comprendre la logique du fonds et l'adapter à son histoire et à son utilisation future. Ainsi, tous les supports sont séparés, rangés par pays puis par reportage. Le numéro d'inventaire donne des informations sur le sujet (auteur, lieu, date de prise de vue) et est formé de sorte que si le fonds est amené à s'enrichir de nouveaux documents, ceux-ci puissent s'insérer sans peine dans le fonds déjà constitué. À titre d'exemple, «PC_SLV_1980_03 » se décompose ainsi :

- PC, pour « Patrick Chauvel ». L'auteur est identifié par ses initiales pour que les fonds d'autres photographes puissent être intégrés au fonds principal si la structure s'étoffe.

- SLV, correspond au code ONU du pays concerné. Ici, le Salvador.

- 1980, date du reportage. Si un reportage se déroule sur plusieurs années, c'est la date de début du reportage qui est indiquée.

- Le numéro à 2 chiffres est le numéro attribué au reportage en question.

Ainsi, chaque numéro est propre à un reportage, quel qu'en soit le support.

Les images qui ont été numérisées possèdent un numéro qui reprend la cote originale du reportage, auquel s'ajoute une lettre précisant la nature de l'objet à partir duquel la numérisation a été faite : $\mathbf{T}$ pour tirage, $\mathbf{D}$ pour diapositive, Ne pour négatif et $\mathbf{N u}$ pour les numériques d'origine. Ce qui donne par exemple : «PC_SLV_1980_03_23_Ne» pour une numérisation d'après négatif.

L'objectif a été d'établir un plan de classement compatible avec l'histoire de ces archives et ce qu'elles nous disent afin d'en dégager des pistes de réflexion préalables à une valorisation appropriée et à l'ouverture du fonds aux chercheurs (fig. 4). 


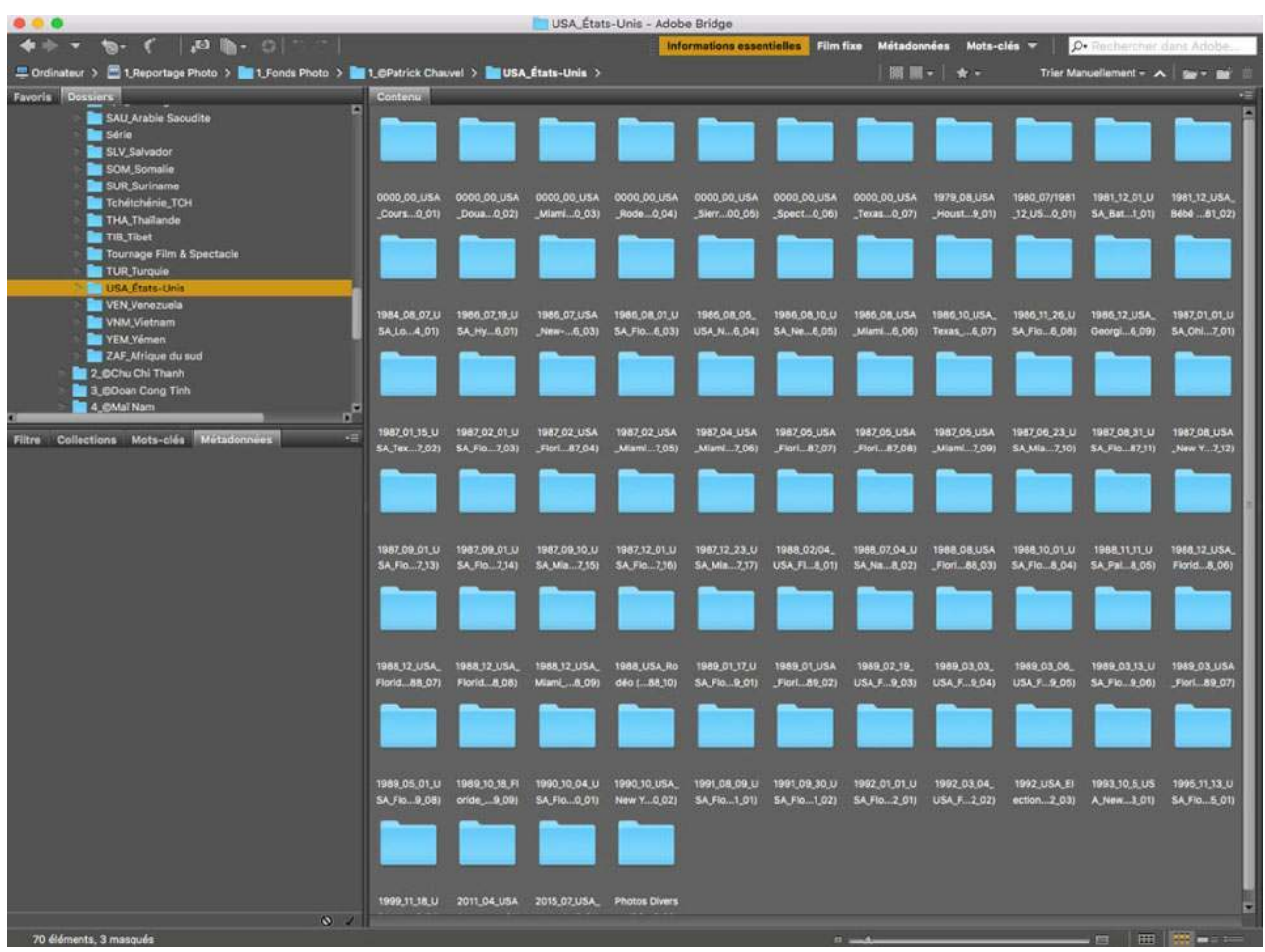

Vue des dossiers numériques après rangement. Ici les dossiers correspondant aux reportages des États-Unis.

(C) Association de préfiguration de la Fondation Patrick Chauvel.

\section{Editing et numérisation}

Pendant près de cinquante ans, Patrick Chauvel a transporté son appareil sur des dizaines de théâtres d'opérations pour fournir à son agence, Sygma, des images témoignant des soubresauts du monde. Aussitôt parvenus à l'agence, les reportages étaient passés au crible et seules les quelques photographies auxquelles les rédacteurs avaient attribué un point rouge étaient retenues pour être immédiatement diffusées dans la presse, alimentant les news aux quatre coins du monde ; les autres, les plus nombreuses, les « non choix », représentant environ $80 \%$ des reportages, étaient instantanément archivés. Sur les 200000 photographies conservées par Corbis, 1200 seulement avaient été numérisées, quantité négligeable.

Le travail engagé à l'Association de préfiguration est tout autre. Les reportages conservés retrouvent leur intégrité pour devenir des archives patrimoniales qui rendent compte à la fois de pratiques professionnelles (des photographes, des agences...), ou de certains usages de la photographie en lien avec un besoin éditorial ou de presse. Ainsi reconsidéré et patrimonialisé, le fonds de photographies de Patrick Chauvel reflète aussi la pratique photographique de son auteur, son regard singulier sur un monde dont il a accompagné les temps forts.

Considérant son œuvre, le photographe a pu, dans le cadre de son projet, dans un autre contexte que celui déterminé par son utilité immédiate, la revisiter. L'existence de la photographie de presse est inextricablement liée à l'événement qu'elle fixe, immédiatement diffusé. Les temps sont de plus en plus courts entre l'editing et la diffusion 
des images. Le but a donc été de poser un nouveau regard sur ces images, saisies il y a plusieurs années.

Après que les photographies ont été inventoriées, une sélection a été numérisée pour être rendue accessible au public. Supervisé par le photographe, un nouvel editing a donc été réalisé, témoignant d'une archive toujours vivante. À côté des images connues, dont certaines sont devenues iconiques, des photographies non connues, voire inédites, sont apparues, éclairant de manière plus distanciée les histoires qu'elles racontent. La sélection d'hier est complétée par celle d'aujourd'hui, celle de l'agence par celle du photographe ou de l'archiviste.

\section{Indexation}

Souvent négligée et parfois jugée inutile, l'indexation est pourtant essentielle. Son rôle est de donner un accès thématique et élargi aux archives tout en préservant le principe $\mathrm{du}$ respect des fonds. L'indexation contribue ainsi à la valorisation des contenus documentaires et historiques, en permettant d'accéder rapidement à une information ponctuelle.

Toutes les images numériques, numérisées et natives, ont été indexées en français et en anglais. Des logiciels de traitement d'images comme Bridge ou Lightroom ont été utilisés pour réaliser l'indexation nécessaire à la traçabilité de ces dernières. Les informations indexées dans les images numériques ont été reportées sur des fiches placées dans les dossiers physiques des reportages contenant des images numérisées.

Ce travail permet de disposer d'un fonds d'archives renseigné et précis avec, pour chaque photo, une "carte d'identité » qui indique la source de diffusion légale et officielle et permet de garantir les droits et la paternité de l'auteur.

Les champs d'indexation des images (titre, description, mots-clés, code ISO pays, auteur, mentions droit d'auteur et source des images, etc.) sont appliqués aux images numériques par reportage. Il n'y a pas d'indexation à la pièce, ce qui aurait demandé trop de temps.

Le lien entre les différents éléments d'un même reportage est créé dans l'instrument de recherche au niveau du reportage, ou du pays lorsqu'il s'agit d'un article concernant un conflit spécifique réunissant plusieurs images extraites de reportages distincts (fig. 5).

Figure 5

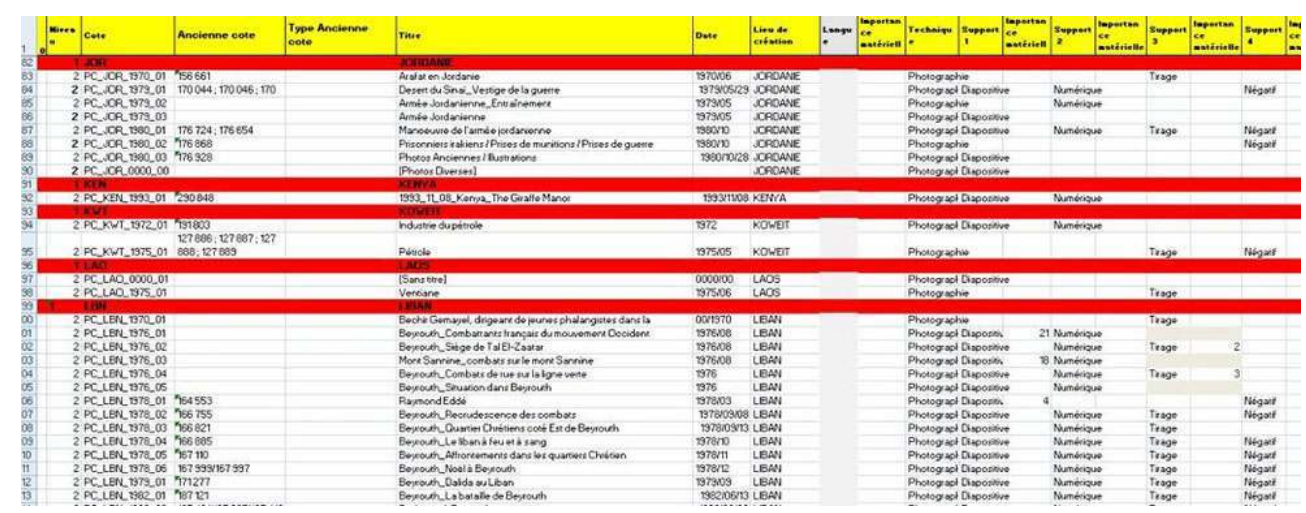

Extrait de l'instrument de recherche créé sur Excel.

(c) Association de préfiguration de la Fondation Patrick Chauvel. 
Le travail effectué sur le fonds du reporter, près de 350000 images tous supports confondus, est considérable. Au fur et à mesure du traitement, il a été nécessaire de redonner leur véritable place aux images et de rétablir les informations les concernant, trop souvent fausses ou modifiées pour répondre à d'autres besoins notamment ceux de la construction des reportages.

Aujourd'hui, après avoir reconstitué le travail du reporter, l'association souhaite aller plus loin, à la fois dans la valorisation du travail photographique mais aussi du travail archivistique effectué, notamment avec la rédaction d'un guide des archives destiné à servir de vade-mecum pour d'autres fonds de ce type. Des expositions sont prévues et l'association envisage de mettre en place des ateliers scolaires et d'ouvrir ses archives au public et aux chercheurs. La reconstitution du fonds a été un début mais il reste encore beaucoup à faire " pour que les peuples n'oublient pas leur passé et ainsi ne soient plus condamnés à le revivre $»^{21}$.

\section{Conclusion}

« Pour les archivistes, l'essentiel n'est pas que l'on puisse toujours écrire l'histoire à chaud, c'est qu'on ne soit pas privé à jamais de l'écrire ${ }^{22}$. "

Nous ne pouvons que souscrire à ces propos de Jean Favier, ancien directeur des Archives nationales. Ce qui est vrai pour une agence travaillant sous pression pour produire de l'information " exclusive » ou pour un quotidien dont les choix sont dictés par l'espace accordé à l'information sur des critères d'urgence, de pays et d'intérêt des lecteurs, ne l'est pas forcément pour le photographe qui travaille à l'information sur le terrain. Les impératifs commerciaux, les contraintes d'une certaine forme d'urgence de la part des agences ne leur permettent pas toujours de juger correctement de la qualité et de la valeur d'une image ou d'une série de photographies. Ainsi, la possibilité qui a été offerte à Patrick Chauvel de créer une structure originale qui rassemble son œuvre de reporter depuis une cinquantaine d'années lui donne-t-elle l'occasion de susciter un nouvel intérêt pour l'information de première main et d'approfondir la connaissance que nous avons des conflits de la seconde moitié du $\mathrm{xx}^{\mathrm{e}}$ siècle.

Le traitement des archives de Patrick Chauvel s'appuie sur les méthodes archivistiques classiques qui ont été adaptées à un fonds aussi particulier que peut l'être celui d'un photoreporter de guerre. Quand on le découvre, on suit le reporter au cours de sa quête de l'information à travers le monde. Et avec cinquante ans de carrière, près de soixantedix pays traversés et plus de mille reportages réalisés, c'est une photothèque d'une grande richesse, précieuse source d'informations, qui a été constituée, organisée et indexée. Elle est prête à être exploitée et peut intéresser tous ceux qui travaillent dans des domaines aussi divers que la recherche, l'histoire, la presse, le documentaire, l'art, la photographie, la polémologie, etc.

L'ambition est d'ajouter à ce fonds photographique les vidéos, les écrits et objets qui apportent un complément d'information important, donnant une contextualisation du métier de reporter sur le terrain et de l'aventure d'un homme, derrière le regard du photographe. La priorité étant la mémoire collective, ce fonds a été identifié et légendé pour informer et documenter les conflits et événements de l'histoire mondiale. 


\section{NOTES}

1. - Citation tirée de la présentation de l'Association de préfiguration de la Fondation Patrick Chauvel, 2014. Voir le site : http://www.fonds-patrickchauvel.com/ [consulté le 04/09/2018].

2. - CHAUVEL, Patrick. Rapporteur de guerre [2003]. Paris : J'ai lu, 2011, p. 6.

3. - D'après Patrice de La Tour du Pin (1911-1975), "Enfants de septembre ", dans La Quête de la joie (1933).

4. - Ibid., p. 317.

5. - Pierre Schoendorffer (1928-2012), cinéaste et écrivain; Joseph Kessel (1898-1979), grand reporter et romancier; Lucien Bodard (1914-1998), écrivain et journaliste; Gilles Caron (1939-1970), photographe et reporter de guerre.

6. - CHAUVEL, Patrick et NOVAT, Antoine. Rapporteur de guerre. Scherkhan production/Canal+, 1998, 53 min.

7. - Au cours de sa longue carrière, Patrick Chauvel a été blessé plusieurs fois, échappant de peu à la mort, mais aussi enlevé, emprisonné et interrogé.

8. - «Massacre du dimanche des Rameaux », mars 1980, faisant suite aux obsèques de Mgr Romero, assassiné.

9. - Biographie de Patrick Chauvel, voir le site: http://www.fonds-patrickchauvel.com/? page_id=9 [consulté le 14/05/2018].

10. - CHAUVEL, Patrick. Rapporteur de guerre. Paris : Oh ! Éditions, 2003.

11. - Voir le site : http://www.warmfoundation.org/ [consulté le 04/09/2018].

12. - Titre du livre (Paris : Les Arènes, 2014) et de l'exposition qui leur ont été consacrés.

13. - Création annoncée par Daniel Barroy, ancien chef de la Mission de la photographie, lors d'une conférence du festival « Visa pour l'image » de Perpignan.

14. - Présentation de la Fondation. Voir le site : http://www.henricartierbresson.org/fondation/ [consulté le 04/09/2018].

15. - «Les seules fondations qui puissent se construire c'est avec la chaleur humaine », Henri Cartier-Bresson, Paris le 11 mai 2004.

16. - Présentation de la Fondation. Voir le site: http://fondationgillescaron.org/ [consulté le 04/09/2018].

17. - Propos de Rémy Ourdan, fondateur du collectif WARM, recueillis par Laurent Geslin pour Le Courrier des Balkans en avril 2013, voir sur le site : http://www.warmfoundation.org/news/lafondation-warm-et-la-memoire-des-conflits-contemporains-le-courrier-des-balkans [consulté le 18/10/2017].

18. - Ibid.

19. - PUECH, Michel. "Corbis-VCG-Getty. Que devient le fonds photo de l'agence Sygma?", 9 février 2016. Voir le site: http://www.a-l-oeil.info/blog/2016/02/09/corbis-vcg-getty-quedevient-le-fonds-photo-de-lagence-sygma/ [consulté le 27/05/2018].

20. - Les IPTC sont les informations concernant l'image : légende, description, mots clés, etc. Ces informations font partie des métadonnées des images.

21. - Winston Churchill : " Un peuple qui oublie son passé est condamné à le revivre ».

22. - FAVIER, Jean. "Constitution et fonction des archives». Dans Académie universelle des cultures, BARRET-DUCROCQ, Françoise (éd.). Pourquoi se souvenir ?. Paris : Grasset, 1999, p. 63. 


\section{RÉSUMÉS}

Face à l'engouement spéculatif que suscitent les archives photographiques de presse sur le marché de l'art, ou à l'inverse, au désintéressement des organes de presse qui s'en délaissent volontiers car elles sont devenues trop encombrantes, il y a ceux que le sujet concerne directement: les reporters-photographes. Patrick Chauvel (né en 1949), reporter de guerre depuis près de cinquante ans, a décidé de créer une structure qui sauvegarde, renseigne et diffuse sa propre production, qui constitue un ensemble patrimonial d'une grande valeur historique, afin de donner accès à des sources d'information et de recherche qui couvrent près d'un demi-siècle d'histoire mondiale. Le défi principal a été d'associer les compétences de trois métiers différents afin de mettre en place un langage commun permettant la compréhension et le dialogue entre les divers domaines concernés que sont la presse, la photographie et les archives. Pour cela, la première étape consiste à récupérer les divers documents (négatifs souples, diapositives, tirages argentiques, objets, carnets de notes, coupures de presse, films, fichiers numériques, etc.) qui se trouvent en plusieurs endroits, notamment dans les fonds Corbis-Sygma et France-Soir, mais aussi dans les affaires personnelles du reporter. Il faut ensuite effectuer le tri des documents par support et par pays, afin de respecter l'histoire de la production, puis les classer par reportages. Afin d'instaurer une certaine continuité dans l'utilisation et la valorisation actuelle du fonds nouvellement reconstitué, il a fallu procéder à un examen général de l'ensemble avant d'entreprendre la numérisation des photos emblématiques et représentatives. Une indexation a été nécessaire pour guider la recherche dans cette documentation sur l'histoire mondiale. La structure mise sur pied par l'Association de préfiguration de la fondation Patrick Chauvel a pour objectif de susciter ce type d'initiatives auprès d'autres reporters et par la suite, d'assurer la sauvegarde et la valorisation de ces fonds si particuliers qui représentent très souvent le travail d'une vie.

Today, press photos can inspire considerable speculative excitement on the art market, whilst at the same time, the major press organs are now abandoning their photo collections because they take up too much room. Between these two extremes are the figures who are the most directly concerned, the photo-reporters. Patrick Chauvel, who was born in 1949, has been an independent war photographer for over fifty years. He has decided to create a structure that will serve to safeguard and diffuse his own work which represents a heritage of considerable historic value, source material for research on nearly half a century of world history. The main challenge was to bring together skills from three different fields of expertise-the press, photography and archives-and to create a common language allowing for dialogue and understanding between them. The first step was simply to gather together the various documents (negatives, slides, traditional prints, objects, notebooks, press cuttings, films, digital files, etc.) which were to be found in several locations, in the collections of Corbis-Sygma and the newspaper France-Soir but also in the reporter's personal affairs. It was then necessary to sort these items by medium and by country, in order to respect the history of their production, and then to classify them by reportage. To create a certain coherence in the use and exploitation of the collection thus reconstituted, an overview was necessary to choose the emblematic pictures and the representative ones to digitise. An index was necessary too, to guide researches in this documentation covering world history. The structure created by the association for the Patrick 
Chauvel foundation hopes to inspire similar initiatives on the part of other photo-reporters and photojournalists, and to help preserve and render useful these collections which often represent a lifetime's work.

INDEX

Mots-clés : archives, archives de presse, archives photographiques, archives photographiques de presse, archivage, agences de presse, fondations, histoire, histoire de l'Humanité, histoire des médias, journalisme, mémoire, Patrick Chauvel, photojournalisme, photographie, presse, reporter

Keywords : archives, press archives, photo archives, press photo archives, archiving, press agency, foundations, history, history of humanity, history of the press, journalism, memory, Patrick Chauvel, photojournalism, photography, press, reporter

\section{AUTEUR}

\section{ÉMILIE FROMENTÈZE}

Archiviste, Doctorante CHCSC - UVSQ / Labex Patrima fromenteze.emilie@gmail.com 